\title{
芳香胺盐酸盐/硼烷体系催化的氢胺化/还原反应研究
}

\author{
张露文＼cjkstart温志国Ｂorzov, Maxim＼cjkstart聂万丽* \\ (乐山师范学院化学学院 天然产物化学与小分子催化四川省高校重点实验室 乐山 614000)
}

\begin{abstract}
摘要 近年来有关受限路易斯酸碱对(FLPs)化学的研究受到了国内外的广泛关注, 但有关芳香胺类 FLPs 的应用研究却 极少涉及. 本工作以硅烷作为还原剂, 路易斯酸三(五氟苯基)硼(BCF)作为催化剂, 用芳香胺盐酸盐代替苯胺, 可一步 反应实现炔烃与苯胺的催化氢胺化还原反应. 研究发现, 取代基较多的三乙基硅烷在反应中表现出较高的反应活性, 吸电子取代基取代的端基芳炔的转化率也较给电子取代基取代的端基芳炔的转化率高. 对催化反应的机理研究表明, 胺盐与 $\mathrm{B}\left(\mathrm{C}_{6} \mathrm{~F}_{5}\right)_{3}$ 及硅烷反应所生成的嗍氢化芳胺盐活性中间体 “ $\left[\mathrm{Ar}_{2} \mathrm{NH}_{2}\right]^{+}\left[\mathrm{H}-\mathrm{B}\left(\mathrm{C}_{6} \mathrm{~F}_{5}\right)_{3}\right]^{-}$” 的产生和分解速度决定着中 间产物亚胺的生成和还原.
\end{abstract}

关键词＼cjkstart受限路易斯酸碱对；芳香胺盐酸盐；三(五氟苯基)硼; 硅烷; 氢胺化/还原反应

\section{Research of $\mathrm{B}\left(\mathrm{C}_{6} \mathrm{~F}_{5}\right)_{3} /$ Aromatic Ammonium Chloride Systems Catalyzed Hydroamination/Reduction Reaction}

\author{
Zhang, Luwen Wen, Zhiguo Borzov, Maxim Nie, Wanli* \\ (Sichuan Province Key Laboratory of Natural Products and Small Molecule Synthesis, Chemical Department of Leshan \\ Normal University, Leshan 614000)
}

\begin{abstract}
Although in recent years the frustrated Lewis pairs (FLPs) reactivity towards small molecule activation has been widely concerned, the reports on the FLPs derived from aromatic amines are few. This paper describes a new method of an one-pot hydroamination/reduction reaction of terminal alkynes with aromatic amines catalyzed by the $\mathrm{B}\left(\mathrm{C}_{6} \mathrm{~F}_{5}\right)_{3} /$ aromatic ammonium chloride systems with a hydridosilane as a source of the hydride. We consider that the active intermediate $\left[\mathrm{Ar}_{2} \mathrm{NH}_{2}\right]^{+}\left[\mathrm{H}-\mathrm{B}\left(\mathrm{C}_{6} \mathrm{~F}_{5}\right)_{3}\right]^{-}$which formed by the aromatic ammonium chloride/ $\mathrm{B}\left(\mathrm{C}_{6} \mathrm{~F}_{5}\right)_{3}$ reaction with silanes plays a very important role on the formation and reduction of the mediate product imines. The hydroamination reaction is firstly induced by the trace amount amines produced by the dissociation of the borohydride aromatic amine salt, which then reacts with the alkynes and forms the imines. Then the borohydride intermediate $\left[\mathrm{Ar}_{2} \mathrm{NH}_{2}\right]^{+}\left[\mathrm{H}-\mathrm{B}\left(\mathrm{C}_{6} \mathrm{~F}_{5}\right)_{3}\right]^{-}$reduces the imines to amines. It has been proved that the borohydride intermediate $\left[\mathrm{Ar}_{2} \mathrm{NH}_{2}\right]^{+}\left[\mathrm{H}-\mathrm{B}\left(\mathrm{C}_{6} \mathrm{~F}_{5}\right)_{3}\right]^{-}$could successfully reduce the corresponding imines to amines in an in-situ reaction condition. However it has been found that the usually most active mono-substituted hydridosilane, such as $\mathrm{PhSiH}_{3}$ shows the poorest reactivity in this case. And the less active trisubstituted silanes such as $\mathrm{Et}_{3} \mathrm{SiH}$ or $\mathrm{Ph}_{3} \mathrm{SiH}$ exhibit the highest reactivity. To explain this abnormal phenomenon the different reaction speeds of the cascade hydroamination/reduction reaction and the dissociation of the borohydride aromatic amine salt should be concerned. Since the dissociation of $\left[\mathrm{Ar}_{2} \mathrm{NH}_{2}\right]^{+}\left[\mathrm{H}-\mathrm{B}\left(\mathrm{C}_{6} \mathrm{~F}_{5}\right)_{3}\right]^{-}$to $\mathrm{H}_{2}$ is comparably quicker than the hydroamination reaction. By reacting with the less active trisubstituted silanes could not only slow down the formation and dissociation of $\left[\mathrm{Ar}_{2} \mathrm{NH}_{2}\right]^{+}$ $\left[\mathrm{H}-\mathrm{B}\left(\mathrm{C}_{6} \mathrm{~F}_{5}\right)_{3}\right]^{-}$, but could also let the hydroamination and reduction steps proceeded completely. Moreover by slowly adding the diluted hydrosilanes to the reaction systems could also improve the reaction. The reaction yield is affected by the substituent on the terminal alkynes, too. The alkynes with the electron withdrawn group show comparably higher reactivity than with the electron donating ones.
\end{abstract}

Keyword frustrated Lewis pairs; aromatic ammonium chloride; $\mathrm{B}\left(\mathrm{C}_{6} \mathrm{~F}_{5}\right)_{3}$; silanes; hydroamination/reduction reaction

\section{1 引言}

氢胺化反应(Hydroamination)通常是将 $\mathrm{N}-\mathrm{H}$ 键加 成到碳碳 $\pi$ 键用来制备各种含 $\mathrm{C}-\mathrm{N}$ 键仲胺或叔胺化合 物的反应. 由于其原子利用率为 $100 \%$, 因此氢胺化反 应在有机合成中也是一类非常重要的反应 ${ }^{[1 \sim 3]}$. 目前有
关氢胺化反应的文献报道, 较多集中在炔烃与苯胺类的 两步法催化氢胺化/还原反应(Hydroamination/reduction) 的研究. 如图 1(A)所示, 其中所涉及的催化剂多为过渡

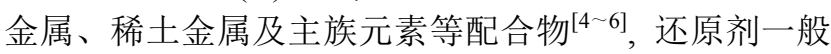
为氢气、氢化硅烷或四氢铝锂等. 近些年, 以大位阻路

\footnotetext{
*E-mail: niew1126@126.com

Received April 6, 2017; published May 23, 2017.
}

Supporting information for this article is available free of charge via the Internet at http://sioc-journal.cn.

Project supported by the National Natural Science Foundation of China (No. 21542011) and Scientific Research Fund of Leshan Normal University (Nos. Z1414, Z1308).

国家自然科学基金(No. 21542011)和乐山师范学院科研(Nos. Z1414, Z1308)资助项目. 
易斯酸 $\mathrm{B}\left(\mathrm{C}_{6} \mathrm{~F}_{5}\right)_{3}$ 与大位阻有机碱(如 $\mathrm{Ph}_{3} \mathrm{P}, t-\mathrm{Bu}_{3} \mathrm{P}$ or TMP 等)所组成的受限路易斯酸碱对(FLPs)为代表的非金属 催化剂对小分子的活化及催化反应研究备受关注 ${ }^{[7 \sim 13]}$. 但有关芳香胺类 FLPs 的应用研究却极少涉及. 2013 年, 加拿大科学家 Stephan 课题组发表了一篇关于利用 $\mathrm{B}\left(\mathrm{C}_{6} \mathrm{~F}_{5}\right)_{3}$ 作为催化剂, 催化苯胺与炔烃氢胺化反应的文 章. 该文利用苯胺 $/ \mathrm{B}\left(\mathrm{C}_{6} \mathrm{~F}_{5}\right)_{3}$ 体系对 $\mathrm{H}_{2}$ 的活化特点, 首次 实现了非金属催化的炔烃的氢胺化/还原的一锅两步合 成方法 ${ }^{[14]}$, 如图 1(B)所示. 2015 年, Stephan 课题组同样 利用 $\mathrm{B}\left(\mathrm{C}_{6} \mathrm{~F}_{5}\right)_{3}$ 作为催化剂, 氢气作为还原剂, 实现了分 子内的一锅两步法氢胺化/还原制备含氮杂环类化合 物 $^{[15]}$, 但是该类反应需要较高的反应温度和压力.

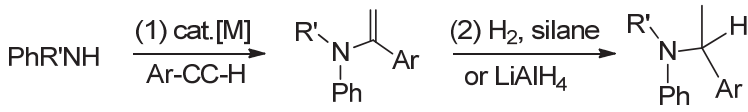

$$
\begin{aligned}
& M=\ln , \mathrm{Zr}, \mathrm{Ti}, \mathrm{Au} \text {, etc. }
\end{aligned}
$$

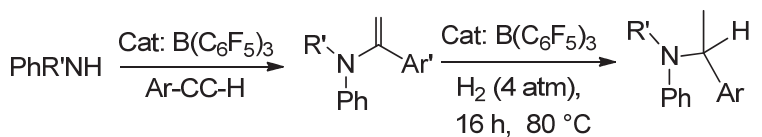

图 1 炔烃与芳胺的氢胺化/还原反应

Figure 1 Reductive hydroamination of phenylacetylenes with amines

我们课题组最近报道了一种研究受限路易斯酸碱 对化学的新方法, 以有机胺盐酸盐代替常规 FLPs 体系 中的烷基胺路易斯碱, 与三(五氟苯基)硼 $\mathrm{B}\left(\mathrm{C}_{6} \mathrm{~F}_{5}\right)_{3}$ 所组 成的新的 “路易斯酸/胺盐” 反应体系, 在以氢化硅烷替 代氢气作为氢源时，可制备各种取代的烷基胺类 “嗍氢 化烷胺盐” ，如图 2(A)所示. 该 “嗍氢化胺盐 $\left[\mathrm{R}_{2} \mathrm{NH}_{2}\right]^{+}$ $\left[\mathrm{H}-\mathrm{B}\left(\mathrm{C}_{6} \mathrm{~F}_{5}\right)_{3}\right]^{-}$” 被认为是还原反应的活性中间体. 研究 表明 “路易斯酸/胺盐” 体系在对醛酮、炔烃、亚胺以及 二氧化碳等的还原反应中均表现出了独特的反应活 性 ${ }^{[16 \sim 18]}$.

由于 “嗍氢化芳胺盐” 的不稳定性, 即使是在低温 下也易释放出氢气的特点，限制了其应用范围. 虽然 2014 年我们以氢化硅烷作为还原剂, 以芳香胺盐酸盐 作为氯代试剂, $\mathrm{BCF}$ 作为催化剂, 首次实现了在温和条

$$
\begin{aligned}
& \underset{\mathrm{R}^{2^{\prime}}}{\mathrm{R}^{1}} \mathrm{NH}_{2} \mathrm{Cl}+\mathrm{R}^{\prime} \mathrm{SiH}_{3} \underset{\mathrm{R}^{\prime} \mathrm{SiH}_{2} \mathrm{Cl}}{\stackrel{\mathrm{B}\left(\mathrm{C}_{6} \mathrm{~F}_{5}\right)_{3}}{\longrightarrow}}\left[\begin{array}{l}
\mathrm{R}^{1} \\
\mathrm{R}^{2-}
\end{array}\right. \\
& \mathrm{R}^{1}, \mathrm{R}^{2}=\mathrm{H}, \text { Alkyl } \\
& \stackrel{\mathrm{R}}{\mathrm{Ph}^{\prime}} \mathrm{NH}_{2} \mathrm{Cl}+\mathrm{R}^{\prime} \mathrm{SiH}_{3} \underset{\mathrm{Cat}_{2}}{\stackrel{\mathrm{B}\left(\mathrm{C}_{6} \mathrm{~F}_{5}\right)_{3}}{-}} \mathrm{R}^{\prime} \mathrm{SiH}_{2} \mathrm{Cl}+\mathrm{R}^{\prime} \mathrm{SiHCl}_{2} \\
& \mathrm{R}=\mathrm{Me}, \mathrm{Ph}
\end{aligned}
$$

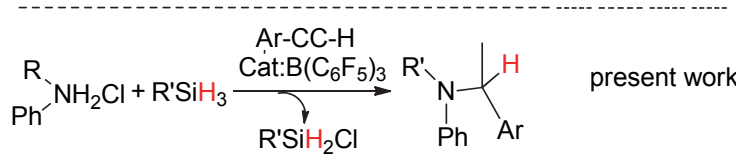

图 $2 \mathrm{~B}\left(\mathrm{C}_{6} \mathrm{~F}_{5}\right)_{3} /$ 有机胺盐酸盐体系参与的反应

Figure 2 The reaction of $\mathrm{B}\left(\mathrm{C}_{6} \mathrm{~F}_{5}\right)_{3} /$ ammonium systems
件下非金属催化的氢化硅烷的氯代，如图 2(B)所示 ${ }^{[19]}$. 但该反应并没有将 “硼氢化芳胺盐” 所释放出的氢气作 为氢源利用.

本文则是以氢化硅烷作为氢源给体, $\mathrm{B}\left(\mathrm{C}_{6} \mathrm{~F}_{5}\right)_{3}$ 为催 化剂, 利用原位产生的“嘲氢化芳胺盐”易于释放出“ $\mathrm{H}_{2}$ ” 特点，实现了在温和反应条件下芳胺盐酸盐与炔烃的一 步法氢胺化/还原反应.

\section{2 结果与讨论}

\section{1 芳香胺盐酸盐/BCF 体系与炔烃的反应研究}

芳香胺盐酸盐可通过向二苯基胺或苯基甲基胺的 二氯甲烷溶液中通入干燥的 $\mathrm{HCl}$ 气体来制备.

表 1 反应所示是在 $\mathrm{B}\left(\mathrm{C}_{6} \mathrm{~F}_{5}\right)_{3}$ 催化下, 不同硅烷还原 二苯胺盐酸盐与炔烃的氢胺化/还原反应. 芳香胺盐酸 盐通常不溶于気代氯仿中, 加入 $\mathrm{B}\left(\mathrm{C}_{6} \mathrm{~F}_{5}\right)_{3}$ 后会缓慢溶解. 硅烷一般应过量并且稀释后慢慢滴加, 随着硅烷加入会 观察到明显的气体产生. 一般情况下 $50{ }^{\circ} \mathrm{C}$ 过夜可使反 应进行完全. 通常我们认为硅烷的反应活性一般为: $\mathrm{PhSiH}_{3}>\mathrm{Ph}_{2} \mathrm{SiH}_{2}>\mathrm{Ph}_{3} \mathrm{SiH}>\mathrm{Et}_{3} \mathrm{SiH}$. 但从表 1 中的数据 可以看出, 在本反应中, 反应的产率却与硅烷活性相反, 三取代的三乙基硅烷 $\mathrm{Et}_{3} \mathrm{SiH}$ 和三苯基硅烷 $\mathrm{Ph}_{3} \mathrm{SiH}$ 表现 出较单取代的苯硅烷更高的反应活性. 而三乙氧基硅烷 参与的反应活性最低. 当炔烃苯基上带有吸电子取代基 时较带有给电子取代基炔烃具有较高的反应活性.

表 1 反应条件的优化 ${ }^{a}$

Table 1 Optimization of the reaction conditions ${ }^{a}$

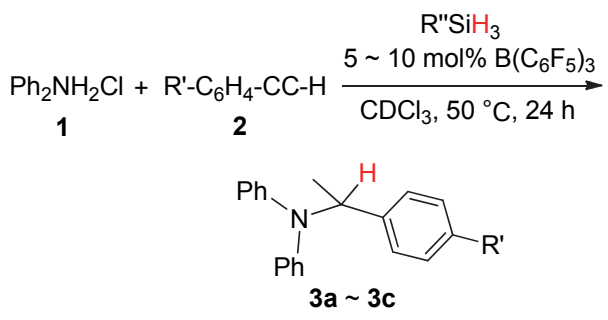

\begin{tabular}{llll}
\hline Entry & Alkyne & Silane & Yield/\% \\
\hline 1 & $\mathrm{C}_{6} \mathrm{H}_{4}-\mathrm{CCH}$ & $\mathrm{Et}_{3} \mathrm{SiH}$ & 3a $62.6^{[20]}$ \\
2 & $P-\mathrm{CH}_{3}-\mathrm{C}_{6} \mathrm{H}_{5}-\mathrm{CCH}$ & $\mathrm{Et}_{3} \mathrm{SiH}$ & 3b $58.6^{[20]}$ \\
3 & $p-\mathrm{F}_{-} \mathrm{C}_{6} \mathrm{H}_{4}-\mathrm{CCH}$ & $\mathrm{Et}_{3} \mathrm{SiH}$ & 3c 100.0 \\
4 & $\mathrm{C}_{6} \mathrm{H}_{4}-\mathrm{CCH}$ & $\mathrm{Ph}_{3} \mathrm{SiH}$ & 3a 70.1 \\
5 & $P-\mathrm{CH}_{3}-\mathrm{C}_{6} \mathrm{H}_{5}-\mathrm{CCH}$ & $\mathrm{Ph}_{3} \mathrm{SiH}$ & 3b 37.6 \\
6 & $p-\mathrm{F}_{-} \mathrm{C}_{6} \mathrm{H}_{4}-\mathrm{CCH}$ & $\mathrm{Ph}_{3} \mathrm{SiH}$ & 3c 82.9 \\
7 & $p-\mathrm{F}_{-} \mathrm{C}_{6} \mathrm{H}_{4}-\mathrm{CCH}$ & $\mathrm{Ph}_{2} \mathrm{SiH}_{3}$ & 3c 60.0 \\
8 & $p-\mathrm{F}-\mathrm{C}_{6} \mathrm{H}_{4}-\mathrm{CCH}$ & $\mathrm{PhSiH}_{3}$ & 3c 38.7 \\
9 & $p$-F-C $\mathrm{C}_{6} \mathrm{H}_{4}-\mathrm{CCH}$ & $(\mathrm{EtO})_{3} \mathrm{SiH}$ & 3c 19.3 \\
\hline${ }^{a}$ NMR Yield; Molar ratio of alkyne to silane $=1: 3$.
\end{tabular}

表 2 中所列出的数据是在苯硅烷的作用下，不同芳 胺与对氟苯乙炔的氢胺化/还原反应. 从图中数据可以 看出，当二芳胺的苯环上对位带有强吸电子取代基 $\mathrm{Br}$ 
时, 反应几乎定量进行(如 $3 \mathbf{d} 、 3 \mathbf{e}$ ); 相反, 当芳胺氮上取 代有烷基时, 反应产率明显降低(如 3f、3g). 化合物 $3 \mathrm{~h}$ 较低的产率说明端基芳炔上的取代基比芳胺上取代基 对反应的影响更显著.

\section{表 2 芳香胺盐酸盐和炔烃的氢胺化/还原反应 ${ }^{a}$}

Table 2 Reductive hydroamination of phenylacetylenes with ammoniums $^{a}$

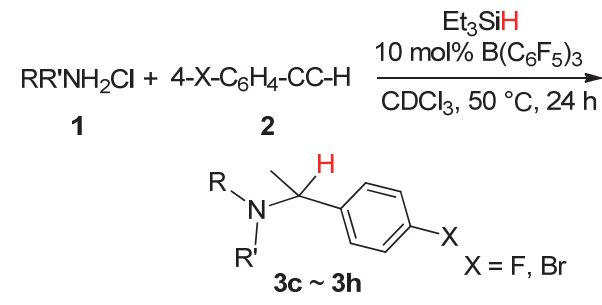

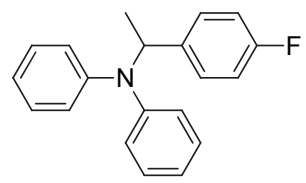

3c, $80.0 \%$

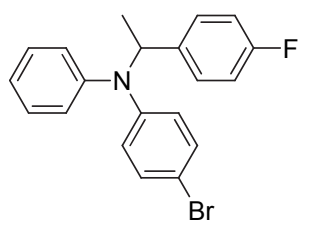

$3 e, 100 \%$

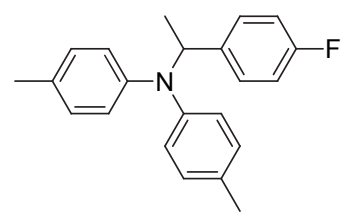

$3 g, 48.1 \%$

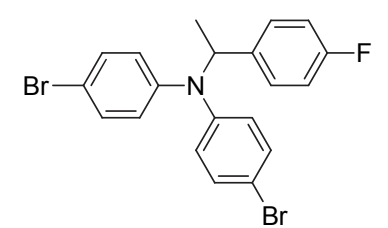

3d, $100 \%$

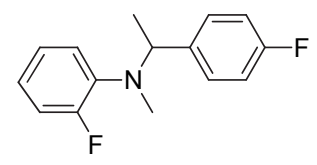

3f, $49.0 \%$

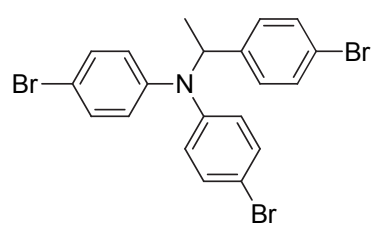

3h, $75.0 \%$
${ }^{a}$ NMR Yield; Molar ratio of alkyne to silane $=1: 1.5$.

对于该反应的反应机理研究, 我们提出了如图 3 所 示的催化氢胺化/还原反应过程. 在 $\mathrm{B}\left(\mathrm{C}_{6} \mathrm{~F}_{5}\right)_{3}$ 催化下, 硅 烷与催化剂 $\mathrm{B}\left(\mathrm{C}_{6} \mathrm{~F}_{5}\right)_{3}$ 及胺盐首先反应形成活性中间体 “嗍氢化芳胺盐 $\left[\mathrm{Ar}_{2} \mathrm{NH}_{2}\right]^{+}\left[\mathrm{H}-\mathrm{B}\left(\mathrm{C}_{6} \mathrm{~F}_{5}\right)_{3}\right]^{-}$”, 少量的硼氢 化芳胺盐分解产生的游离胺 $\mathrm{Ar}_{2} \mathrm{NH}$ 在 $\mathrm{B}\left(\mathrm{C}_{6} \mathrm{~F}_{5}\right)_{3}$ 催化下 与炔烃反应生成亚胺. 这一中间产物亚胺可被活性 “嗍

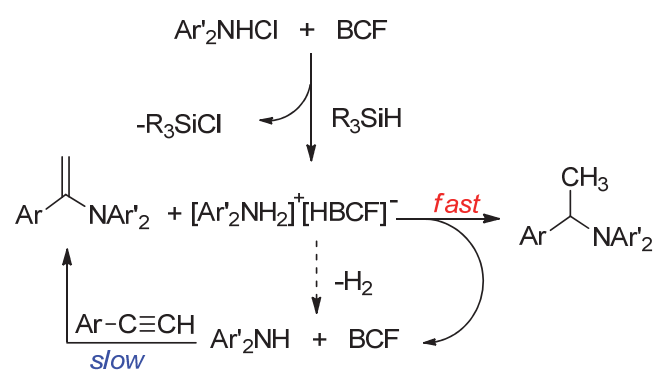

图 3 一步法氢胺化/还原反应机理

Figure 3 Mechanism of the one-step reductive hydroaminations
氢化芳胺盐” 还原再释放出游离的胺 $\mathrm{Ar}_{2} \mathrm{NH}$ 及 $\mathrm{B}\left(\mathrm{C}_{6} \mathrm{~F}_{5}\right)_{3}$ 使得催化循环可以继续.

而决定 $\mathrm{B}\left(\mathrm{C}_{6} \mathrm{~F}_{5}\right)_{3}$ 催化下, 硅烷参与的氢胺化/还原反 应产率的主要因素是所形成的活性中间体 “嗍氢化芳胺 盐” 的分解速度. 由于炔烃的氢胺化反应较亚胺中间体 的还原反应速度慢的特点, 活性较高的硅烷与 $\mathrm{B}\left(\mathrm{C}_{6} \mathrm{~F}_{5}\right)_{3}$ 及胺盐形成中间体 “ $\left[\mathrm{R}_{2} \mathrm{NH}_{2}\right]^{+}\left[\mathrm{H}-\mathrm{B}\left(\mathrm{C}_{6} \mathrm{~F}_{5}\right)_{3}\right]^{-}$” 的速度较 快, 受限于较慢的氢胺化反应及不稳定的硼氢化芳胺盐 分解的特点, 因此还原产物产率不高. 而活性较低的三 取代硅烷, 由于形成硼氢化芳胺盐的速度较慢, 分解也 就较慢，中间体亚胺的还原较充分，因此产率也较高. 总之, 对于活性较高的硅烷参与的反应，通过稀释或缓 慢加入硅烷的方法来控制嗍氢化芳胺盐的产生速率，可 有效地提高氢胺化/还原反应的产率.

为了对反应机理的合理性进行验证, 我们设计了如 图 4 所示的实验: $\mathrm{B}\left(\mathrm{C}_{6} \mathrm{~F}_{5}\right)_{3}$ 催化下的芳香胺盐酸盐与烯 胺的还原反应。实验证明，原位产生的 “硼氢化芳胺盐” 可在温和条件下将烯胺还原为胺.

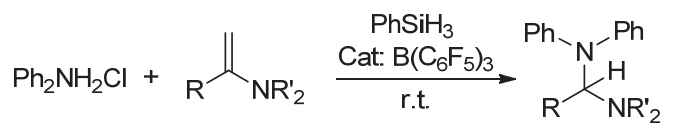

图 $4 \quad B\left(\mathrm{C}_{6} \mathrm{~F}_{5}\right)_{3} /$ 芳香胺盐酸盐体系对亚胺的还原

Figure 4 Reduction of imines by the $\mathrm{B}\left(\mathrm{C}_{6} \mathrm{~F}_{5}\right)_{3} /$ aromatic ammonium system

\section{3 结论}

本文是我们课题组所报道的一系列有关以有机胺 盐酸盐代替常规 FLPs 体系中的路易斯碱, 组成的新的 “路易斯酸/胺盐” 反应体系, 来研究 FLPs 化学的工作之 一。首次实现了原位产生的 “硼氢化芳胺盐” 在炔烃的 氢胺化及还原中的应用. 该反应进一步扩展了有机芳胺 盐体系的应用领域. 尽管以硅烷代替氢气总会不可避免 硅烷副产物的形成，但是以有机胺盐酸盐代替常规 FLPs 体系中的路易斯碱，所组成的新的 “路易斯酸/胺 盐” 反应体系已显示出的独特反应活性和特征，促使我 们有必要继续对这一新的研究方法进行深入的探索研 究.

\section{4 实验部分}

实验所有操作都是在无水无氧条件下进行的，所有 反应溶剂及氛代试剂均是在氮气保护下经过干燥除水 处理.

\section{1 芳香胺盐酸盐的制备}

分别将 $5.0 \mathrm{~mL}$ 或者 $5.0 \mathrm{~g}$ 的芳香胺和 $10.0 \mathrm{~mL}$ $\mathrm{CH}_{2} \mathrm{Cl}_{2}$ 加入 Schlenk 瓶中, 搅拌下通入干燥的 $\mathrm{HCl}$ 气体, 室温搅拌反应 $5 \mathrm{~h}$. 在反应过程中大部分的盐酸盐会沉 淀析出, 过滤, $\mathrm{CH}_{2} \mathrm{Cl}_{2}$ 洗涤 3 次, 真空抽滤干燥过夜可 
得到较纯净的盐酸盐. 用此方法制备二苯胺盐酸盐、双 (4-溴苯基)胺盐酸盐、 $N$-甲基-2 氟苯胺盐酸盐、4-溴- $N$ 苯基胺盐酸盐.

\section{$4.2 \quad N$-(1-(4-fluorophenyl)ethyl)- $N$-(phenyl)benzen- amine (3c)的制备}

在氮气保护下, 将 $205.0 \mathrm{mg}(1.0 \mathrm{mmol})$ 二苯胺盐酸 盐置于反应瓶中, 然后加入 $41.0 \mathrm{mg}(0.1 \mathrm{mmol})$ 三(五氟 苯基)硼于反应瓶中, 用 $9.0 \mathrm{~mL}$ 氯仿溶解, 再加入 120.0 $\mathrm{mg}(1.0 \mathrm{mmol})$ 对氟苯乙炔, 最后缓慢加入氯仿稀释 10 倍的 $180.0 \mathrm{mg}(1.5 \mathrm{mmol})$ 三乙基硅烷, $50{ }^{\circ} \mathrm{C}$ 下反应 $24 \mathrm{~h}$. 硅胶过柱提纯分离[洗脱液为 $V$ (石油醚) : $V$ (乙酸乙酯) $=6: 1]$, 分离产率为 $51.5 \% .{ }^{1} \mathrm{H} \mathrm{NMR}\left(400 \mathrm{MHz}, \mathrm{CDCl}_{3}\right.$, $298 \mathrm{~K}) \delta: 1.49$ (d, $\left.J=7.01 \mathrm{~Hz}, 3 \mathrm{H}, \mathrm{CH}_{3}-\mathrm{CH}\right), 5.29$ (q, $J=$ $\left.6.95 \mathrm{~Hz}, 1 \mathrm{H}, \mathrm{CH}_{3} \mathrm{CH}\right), 6.83(\mathrm{~d}, J=8.52 \mathrm{~Hz}, 4 \mathrm{H}, o-\mathrm{Ph})$, $7.01 \sim 6.92(\mathrm{~m}, 4 \mathrm{H}, p-\mathrm{Ph} / \mathrm{F}-\mathrm{Ph}), 7.20(\mathrm{t}, J=7.33 \mathrm{~Hz}, 4 \mathrm{H}$, $m$-Ph), $7.34 \sim 7.17$ (m, 2H, F-Ph); ${ }^{13} \mathrm{C}\left\{{ }^{1} \mathrm{H}\right\} \mathrm{NMR}(100$ $\left.\mathrm{MHz}, \mathrm{CDCl}_{3}, 298 \mathrm{~K}\right) \delta: 19.11,56.75,114.96,115.21$, 128.54, 128.62, $122.97(o-\mathrm{Ph}), 121.75(p-\mathrm{Ph}), 129.02$ $(m-\mathrm{Ph}), 139.45\left(\mathrm{~d},{ }^{4} J_{\mathrm{F}-\mathrm{C}}=2.92 \mathrm{~Hz}\right), 146.74,160.45 / 160.91$ $\left(\mathrm{d},{ }^{1} J_{\mathrm{F}-\mathrm{C}}=245.14 \mathrm{~Hz}\right)$; GC-MS (EI) $m / z: 291.16\left(\mathrm{M}^{+}\right) .{ }^{19} \mathrm{~F}$ $\left\{{ }^{1} \mathrm{H}\right\} \mathrm{NMR}\left(376 \mathrm{MHz}, \mathrm{CDCl}_{3}, 298 \mathrm{~K}\right) \delta:-116.23$.

4.3 4-Bromo-N-(1-(4-fluorophenyl)ethyl)- $N-(p-$ bromophenyl)benzenamine $(\mathbf{3 d})$ 的制备

在氮气保护下, 将 $361.5 \mathrm{mg}$ (1.0 mmol)双(4-溴苯 基)胺盐酸盐置于反应瓶中, 然后加入 $41.0 \mathrm{mg}(0.1$ $\mathrm{mmol}$ )三(五氟苯基)硼于反应瓶中, 用 $9.0 \mathrm{~mL}$ 氯仿溶解, 再加入 $120.0 \mathrm{mg}(1.0 \mathrm{mmol})$ 对氟苯乙炔, 最后缓慢加入 氯仿稀释 10 倍的 $180.1 \mathrm{mg}(1.5 \mathrm{mmol})$ 三乙基硅烷, $50{ }^{\circ} \mathrm{C}$ 下反应 $24 \mathrm{~h}$. 硅胶过柱提纯 [洗脱液为 $V($ 石油 醚) : $V$ (乙酸乙酯 $)=6: 1]$, 分离产率为 $46.5 \%$. ${ }^{1} \mathrm{H} \mathrm{NMR}$ $\left(400 \mathrm{MHz}, \mathrm{CDCl}_{3}, 298 \mathrm{~K}\right) \delta: 1.44\left(\mathrm{~d}, 3 \mathrm{H},{ }^{1} J_{\mathrm{CH}}=127.6 \mathrm{~Hz}\right.$, $\left.{ }^{3} J_{\mathrm{HH}}=7.1 \mathrm{~Hz}, \mathrm{CH}_{3}\right), 5.19$ (q, $\left.1 \mathrm{H},{ }^{3} J_{\mathrm{HH}}=7.1 \mathrm{~Hz}, \mathrm{CH}_{3} \mathrm{CH}\right)$, 6.67, $7.27\left(\mathrm{~d}, 8 \mathrm{H},{ }^{3} J_{\mathrm{AX}}=8.9 \mathrm{~Hz}, \mathrm{H} 2,6\right.$ and $\mathrm{H} 3,5$ in $4-\mathrm{BrC}_{6} \mathrm{H}_{4}$, respectively), $6.94 \sim 7.24\left(\mathrm{~m}, 4 \mathrm{H}, 4-\mathrm{FC}_{6} \mathrm{H}_{4}\right)$; ${ }^{13} \mathrm{C}\left\{{ }^{1} \mathrm{H}\right\} \mathrm{NMR}\left(100 \mathrm{MHz}, \mathrm{CDCl}_{3}, 298 \mathrm{~K}\right) \delta: 19.11\left(\mathrm{~s}, \mathrm{CH}_{3}\right)$, $56.99\left(\mathrm{~s}, \mathrm{CH}_{3} \mathrm{CH}\right), 114.98(\mathrm{~s}, \mathrm{CBr}), 115.28\left(\mathrm{~d},{ }^{2} J_{\mathrm{FC}}=21.3\right.$ $\left.\mathrm{Hz}, 4-\mathrm{F}-\mathrm{C}_{6} \mathrm{H}_{4}\right), 124.59,132.08\left(\mathrm{~s}, 4-\mathrm{BrC}_{6} \mathrm{H}_{4}\right), 128.46$ (d, $\left.{ }^{3} J_{\mathrm{FC}}=8.0 \mathrm{~Hz}, 4-\mathrm{F}-\mathrm{C}_{6} \mathrm{H}_{4}\right), 138.47 \quad\left(\mathrm{~d},{ }^{4} J_{\mathrm{FC}}=3.4 \mathrm{~Hz}\right.$, $\left.4-\mathrm{FC}_{6} \mathrm{H}_{4}\right), 145.46\left(\mathrm{~s}, 4-\mathrm{BrC}_{6} \mathrm{H}_{4}\right), 161.76\left(\mathrm{~d},{ }^{1} J_{\mathrm{FC}}=245.7\right.$ $\mathrm{Hz}, \mathrm{CF}) ;{ }^{19} \mathrm{~F}$ NMR $\left(376 \mathrm{MHz}, \mathrm{CDCl}_{3}, 298 \mathrm{~K}\right) \delta:-115.07$ (tt, ${ }^{3} J_{\mathrm{FH}}=8.3 \mathrm{~Hz},{ }^{4} J_{\mathrm{FH}}=5.5 \mathrm{~Hz}$ ).

$4.4 \quad N$-(1-(4-fluorophenyl)ethyl)- $N$-(p-bromophenyl) benzenamine $(3 e)$ 的制备

在氮气保护下, 将 $285.0 \mathrm{mg}(1.0 \mathrm{mmol}) 4$-溴- $N$-苯 基胺盐酸盐置于反应瓶中, 然后加入 $41.0 \mathrm{mg}(0.1$ $\mathrm{mmol}$ )三(五氟苯基)硼于反应瓶中, 用 $9.0 \mathrm{~mL}$ 氯仿溶解, 再加入 $119.8 \mathrm{mg}(1.0 \mathrm{mmol})$ 对氟苯乙炔, 最后缓慢加入
氯仿稀释 10 倍的 $180.2 \mathrm{mg}(1.5 \mathrm{mmol})$ 三乙基硅烷, $50{ }^{\circ} \mathrm{C}$ 下反应 $24 \mathrm{~h}$. 硅胶过柱提纯[洗脱液为 $V($ 石油 醚 $): V($ 乙酸乙酯 $)=6: 1]$, 分离产率为 $40.4 \%$. ${ }^{1} \mathrm{H}$ NMR $\left(400 \mathrm{MHz}, \mathrm{CDCl}_{3}, 298 \mathrm{~K}\right) \delta: 1.46\left(\mathrm{~d}, 3 \mathrm{H},{ }^{1} J_{\mathrm{CH}}=\right.$ $\left.127.6 \mathrm{~Hz},{ }^{3} J_{\mathrm{HH}}=7.0 \mathrm{~Hz}, \mathrm{CH}_{3}\right), 5.22\left(\mathrm{q}, 1 \mathrm{H},{ }^{1} J_{\mathrm{CH}}=136.0\right.$ $\left.\mathrm{Hz},{ }^{3} J_{\mathrm{HH}}=7.0 \mathrm{~Hz}, \mathrm{CH}_{3} \mathrm{CH}\right), 6.60\left(\mathrm{~d},{ }^{3} J_{\mathrm{AX}}=9.1 \mathrm{~Hz}, 2 \mathrm{H}\right.$, $\left.4-\mathrm{Br}-\mathrm{C}_{6} \mathrm{H}_{4}\right), 7.21\left(\mathrm{~d},{ }^{3} J_{\mathrm{AX}}=9.1 \mathrm{~Hz}, 2 \mathrm{H}, 4-\mathrm{BrC}_{6} \mathrm{H}_{4}\right), 6.94 \sim$ $7.26\left(\mathrm{~m}, 4 \mathrm{H},{ }^{3} J_{\mathrm{AX}} \sim{ }^{3} J_{\mathrm{AF}}=8.8 \mathrm{~Hz},{ }^{4} J_{\mathrm{XF}}=5.4 \mathrm{~Hz}, 4-\mathrm{FC}_{6} \mathrm{H}_{4}\right)$, $6.90 \sim 7.23(\mathrm{~m}, 5 \mathrm{H}, \mathrm{Ph}) ;{ }^{13} \mathrm{C}\left\{{ }^{1} \mathrm{H}\right\} \mathrm{NMR}\left(100 \mathrm{MHz}, \mathrm{CDCl}_{3}\right.$, $298 \mathrm{~K}) \delta: 19.34\left(\mathrm{~s}, \mathrm{CH}_{3}\right), 57.01\left(\mathrm{~s}, \mathrm{CH}_{3} \mathrm{CH}\right), 112.97$ (s, $\mathrm{CBr}), 115.21\left(\mathrm{~d},{ }^{2} J_{\mathrm{FC}}=21.3 \mathrm{~Hz}, 4-\mathrm{F}-\mathrm{C}_{6} \mathrm{H}_{4}\right), 122.27$, 131.77, 146.46 (all s, C2, 6, C3, 5 and $\mathrm{C} 1$ in $4-\mathrm{Br}_{-} \mathrm{C}_{6} \mathrm{H}_{4}$, respectively), $129.29\left(\mathrm{~d},{ }^{3} J_{\mathrm{FC}}=8.0 \mathrm{~Hz}, \mathrm{C} 2,6\right.$ in $\left.4-\mathrm{F}-\mathrm{C}_{6} \mathrm{H}_{4}\right)$, 123.54, 125.28, 129.29, 145.82 (all s, C4, C2, 6, C3, 5, and $\mathrm{C} 1$ in $\mathrm{Ph}$, respectively), $138.96\left(\mathrm{~d},{ }^{4} J_{\mathrm{FC}}=3.4 \mathrm{~Hz}, \mathrm{C} 1\right.$ in $\left.4-\mathrm{F}-\mathrm{C}_{6} \mathrm{H}_{4}\right), 161.75\left(\mathrm{~d},{ }^{1} J_{\mathrm{FC}}=245.2 \mathrm{~Hz}, \mathrm{CF}\right) ;{ }^{19} \mathrm{~F}$ NMR $(376$ $\left.\mathrm{MHz}, \mathrm{CDCl}_{3}, 298 \mathrm{~K}\right) \delta:-115.58\left(\mathrm{tt},{ }^{3} J_{\mathrm{FH}}=8.8 \mathrm{~Hz},{ }^{4} J_{\mathrm{FH}}=\right.$ $5.4 \mathrm{~Hz})$.

\subsection{2-Fluoro-N-(1-(4-fluorophenyl)ethyl)- $N$-(methyl)- benzenamine (3f)的制备}

在氮气保护下, 将 $161.6 \mathrm{mg}(1.0 \mathrm{mmol}) \mathrm{N}$-甲基-2 氟 苯胺盐酸盐置于反应瓶中, 然后加入 $41.0 \mathrm{mg}(0.1$ $\mathrm{mmol}$ )三(五氟苯基)硼于反应瓶中, 用 $9.0 \mathrm{~mL}$ 氯仿溶解, 再加入 $119.8 \mathrm{mg}(1.0 \mathrm{mmol})$ 对氟苯乙炔, 最后缓慢加入 氯仿稀释 10 倍的 $179.8 \mathrm{mg}(1.5 \mathrm{mmol})$ 三乙基硅烷, $50{ }^{\circ} \mathrm{C}$ 下反应 $24 \mathrm{~h}$. 硅胶过柱提纯 [洗脱液为 $V$ (石油 醚 $): V($ 乙酸乙酯 $)=6: 1]$, 分离产率为 $35.5 \%$. ${ }^{1} \mathrm{H}$ NMR (400 MHz, $\left.\mathrm{CDCl}_{3}, 298 \mathrm{~K}\right) \delta: 1.43\left(\mathrm{~d},{ }^{1} J_{\mathrm{CH}}=123.7\right.$ $\left.\mathrm{Hz},{ }^{3} J_{\mathrm{HH}}=6.9 \mathrm{~Hz}, 3 \mathrm{H}, \mathrm{CHCH}_{3}\right), 2.49\left(\mathrm{~s}, 3 \mathrm{H},{ }^{1} J_{\mathrm{CH}}=135.1\right.$ $\left.\mathrm{Hz}, \mathrm{NCH}_{3}\right), 4.72\left(\mathrm{q}, 1 \mathrm{H},{ }^{1} J_{\mathrm{CH}}=137.4 \mathrm{~Hz},{ }^{3} J_{\mathrm{HH}}=6.9 \mathrm{~Hz}\right.$, $\left.\mathrm{CH}_{3} \mathrm{CH}\right), 6.99 \sim 7.33\left(\mathrm{~m}, 4 \mathrm{H}, 4-\mathrm{FC}_{6} \mathrm{H}_{4}\right), 6.89 \sim 7.03(\mathrm{~m}$, $\left.2-\mathrm{FC}_{6} \mathrm{H}_{4}\right) ;{ }^{13} \mathrm{C}\left\{{ }^{1} \mathrm{H}\right\} \mathrm{NMR}\left(100 \mathrm{MHz}, \mathrm{CDCl}_{3}, 298 \mathrm{~K}\right) \delta$ : $15.90\left(\mathrm{~s}, \mathrm{CHCH}_{3}\right), 33.31\left(\mathrm{~s}, \mathrm{NCH}_{3}\right), 59.56\left(\mathrm{~d},{ }^{4} J_{\mathrm{FC}}=5.5\right.$ $\left.\mathrm{Hz}, \mathrm{CH}_{3} \mathrm{CH}\right), 114.85\left(\mathrm{~d},{ }^{2} J_{\mathrm{FC}}=21.3 \mathrm{~Hz}, \mathrm{C} 3,5\right.$ in $\left.4-\mathrm{F}-\mathrm{C}_{6} \mathrm{H}_{4}\right), 116.20\left(\mathrm{~d},{ }^{2} J_{\mathrm{FC}}=21.2 \mathrm{~Hz}, \mathrm{C} 3\right.$ in $\left.2-\mathrm{F}-\mathrm{C}_{6} \mathrm{H}_{4}\right)$, $121.56\left(\mathrm{~d},{ }^{3} J_{\mathrm{FC}}=2.8 \mathrm{~Hz}, \mathrm{C} 4\right.$ in $\left.2-\mathrm{F}-\mathrm{C}_{6} \mathrm{H}_{4}\right), 122.01$ (br. d, ${ }^{3} J_{\mathrm{FC}}=7.6 \mathrm{~Hz}, \mathrm{C} 6$ in $\left.2-\mathrm{F}-\mathrm{C}_{6} \mathrm{H}_{4}\right), 124.25\left(\mathrm{~d},{ }^{4} J_{\mathrm{FC}}=3.8 \mathrm{~Hz}\right.$, $\mathrm{C} 5$ in $\left.2-\mathrm{F}-\mathrm{C}_{6} \mathrm{H}_{4}\right), 129.00\left(\mathrm{~d},{ }^{3} J_{\mathrm{FC}}=8.0 \mathrm{~Hz}, \mathrm{C} 3\right.$ in $\left.2-\mathrm{F}-\mathrm{C}_{6} \mathrm{H}_{4}\right), 137.97\left(\mathrm{~d},{ }^{4} J_{\mathrm{FC}}=2.7 \mathrm{~Hz}, \mathrm{C} 1\right.$ in $\left.4-\mathrm{F}-\mathrm{C}_{6} \mathrm{H}_{4}\right)$, 139.94 (br. d, ${ }^{2} J_{\mathrm{FC}}=8.3 \mathrm{~Hz}, \mathrm{C} 1$ in $\left.2-\mathrm{F}-\mathrm{C}_{6} \mathrm{H}_{4}\right), 156.25(\mathrm{~d}$, ${ }^{1} J_{\mathrm{FC}}=244.9 \mathrm{~Hz}, \mathrm{CF}$ in $\left.2-\mathrm{F}-\mathrm{C}_{6} \mathrm{H}_{4}\right), 161.88\left(\mathrm{~d},{ }^{1} J_{\mathrm{FC}}=244.8\right.$ $\mathrm{Hz}, \mathrm{CF}$ in $\left.4-\mathrm{F}-\mathrm{C}_{6} \mathrm{H}_{4}\right) ;{ }^{19} \mathrm{~F}\left\{{ }^{1} \mathrm{H}\right\} \mathrm{NMR}\left(376 \mathrm{MHz}, \mathrm{CDCl}_{3}\right.$, $298 \mathrm{~K}) \delta:-121.86\left(\mathrm{~m}, 2-\mathrm{FC}_{6} \mathrm{H}_{4}\right) ;-116.12(\mathrm{~m}$, $\left.4-\mathrm{FC}_{6} \mathrm{H}_{4}\right)$.

\section{References}

[1] Müller, T. E.; Hultzsch, K. C.; Yus, M.; Foubelo, F.; Tada, M. Chem. Rev. 2008, 108, 3795.

[2] Severin, R.; Doye, S. Chem. Soc. Rev. 2007, 36, 1407. 
[3] Nishina, N.; Yamamoto, Y. Top. Organomet. Chem. 2013, 43, 115.

[4] (a) Sun, Q.; Wang, Y.-R.; Yuan, D.; Yao,Y.-M.; Shen, Q. Organometallics 2014, 33, 994. (b) Liang, S.-Z.; Hammond, L.; Xu, B.; Hammond, G. B. Adv. Synth. Catal. 2016, 358(20), 3313. (c) Sakai, N.; Takahashi, N.; Ogiwara, Y. Eur. J. Org. Chem. 2014, 5078.

[5] Wang, J.; Cui, D.-M. Chin. J. Org. Chem. 2016, 36(6), 1163 (王剑, 崔冬梅, 有机化学, 2016, 36(6), 1163.)

[6] Bian, R.-J.; Bao, X.-G. Chin. J. Org. Chem. 2017, 37(1), 190 (市荣 剑, 鲍晓光, 有机化学, 2017, 37(1), 190.)

[7] McCahill, J. S. J.; Welch, G. C.; Stephan, D. W. Angew. Chem., Int. Ed. 2007, 46, 4968.

[8] Dureen, M. A.; Brown, C. C.; Stephan, D. W. Organometallics 2010, $29,6594$.

[9] Sajid, M.; Elmer, L.-M.; Rosorius, C.; Daniliuc, C. G.; Grimme, S.; Kehr, G.; Erker, G. Angew. Chem., Int. Ed. 2013, 52, 2243.

[10] Mçmming, C. M.; Otten, E.; Kehr, G.; Frçhlich, R.; Grimme, S.; Stephan, D. W.; Erker, G. Angew. Chem., Int. Ed. 2009, 48, 6643.

[11] Cardenas, A. J. P.; Culotta, B. J.; Warren, T. H.; Grimme, S.; Stute, A.; Froehlich, R.; Kehr, G.; Erker, G. Angew. Chem., Int. Ed. 2011, $50,7567$.

[12] Otten, E.; Neu, R. C.; Stephan, D. W. J. Am. Chem. Soc. 2009, 131,
9918.

[13] Sajid, M.; Klose, A.; Birkmann, B.; Liang, L.; Schirmer, B.; Wiegand, T.; Eckert, H.; Lough, A. J.; Froehlich, R.; Daniliuc, C. G.; Grimme, S.; Stephan, D. W.; Kehr, G.; Erker, G. Chem. Sci. 2013, 4, 213.

[14] Mahdi, T.; Stephan, D. W. Angew. Chem., Int. Ed. 2013, 52, 12418.

[15] Mahdi, T.; Stephan, D. W. Chem. Eur. J. 2015, 21, 11134.

[16] Wen, Z.-G.; Tian, C.; Borzov, M.; Nie, W.-L. Acta Chim. Sinica 2016, 74, 498. (温志国, 田冲, Borzov Maxim, 聂万丽, 化学学报, 2016, 74, 498.)

[17] Hu, X.; Tian, C.; Borzov, M.; Nie, W.-L. Acta Chim. Sinica 2015, 73, 1025. (胡茜, 田冲, Borzov Maxim, 聂万丽, 化学学报, 2015, 73, 1025.)

[18] Nie, W.-L.; Sun, G.-F.; Tian, C.; Borzov, M. V. Z. Naturforsch. 2016, $71(10) b, 1029$.

[19] Tian, C.; Jiang, Y.; Borzov, M.; Nie, W.-L. Acta Chim. Sinica 2015, 73, 1203. (田冲, 姜亚, Borzov Maxim, 聂万丽, 化学学报, 2015, 73, 1203.)

[20] Li, L.; Huang, G.; Chen, Z.; Liu, W.; Wang, X.; Chen, Y.; Yang, L.; Li, W.; Li, Y. Eur. J. Org. Chem. 2012, 28, 5564

(Cheng, F.) 